\title{
OBSERVATIONS SUR LA PRODUCTION LAITIÉRE DES TRUIES
}

PAR

\section{E. SALMON-LEGAGNEUR (')}

Station de Recherches sur l'Élevage, C. N. R. Z., Jouy-en Josas (S. et Oise)

L'étude de la production laitière des truies a fait l'objet d'un certain nombre de travaux au cours de ces dernières années.

Après nous être nous-mêmes penchés sur le problème des méthodes de mesure de la production laitière des truies et avoir mis au point une technique de mesure (SALMON-LEGAGNEUR, I956), nous avons pu réunir un certain nombre de résultats, dont nous donnons ici une première analyse.

Ces résultats ont été acquis au cours des années 1954-57 sur notre troupeau expérimental ; ils comportent l'enregistrement de 7 I lactations de truies Yorkshire Large White.

\section{I. - LA LACTATiON DE LA TRUIE}

\section{Production laitière moyenne.}

La production laitière journalière moyenne pour les $7 \mathrm{I}$ lactations a été de $5,3 \mathrm{~kg}$ de lait par truie (pour une moyenne de 8,2 porcelets sevrés par portée). Cette valeur est du même ordre que celles publiées dans les travaux récents. Il est d'ailleurs à remarquer que tous les résultats de ces dernières années sont notablement plus élevés que les premiers trouvés, ce qui s'explique vraisemblablement par une amélioration des techniques de mesure et, en particulier, par un raccourcissement des intervalles de contrôle (tableau I). Pour cette raison, seuls les résultats de ces dix dernières années peuvent donc être comparés valablement entre eux.

La production journalière moyenne la plus élevée que nous ayons enregistrée a été celle d'une truie à sa đeuxième lactation qui a produit $9,64 \mathrm{~kg}$ de lait par jour avec un maximum de $12,6 \mathrm{~kg}$ au milieu de la période de lactation.

(1) Avec la collaboration technique de J. Rettagliati et la participation de G. Gomez, Ingénieur agronome, Lima, Pérou. 


\section{TABIEAU I}

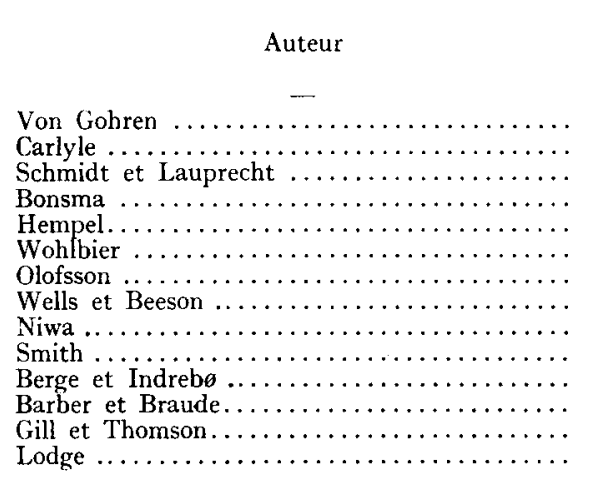

Année

--
1865
1903
1926
1935
1928
1928
1930
1940
1951
1952
1953
1955
1956
1957

Intervalle entre
les contrôles

Lait par truie et par jour (moy.)

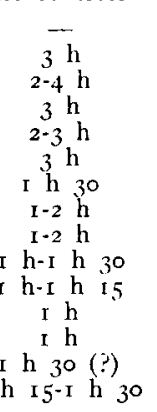

La production laitière totale au cours de la lactation ( 56 jours) a été en moyenne de : $297 \pm 9 \mathrm{I} \mathrm{kg}$ de lait, avec la distribution que nous donnons à la figure $I$.

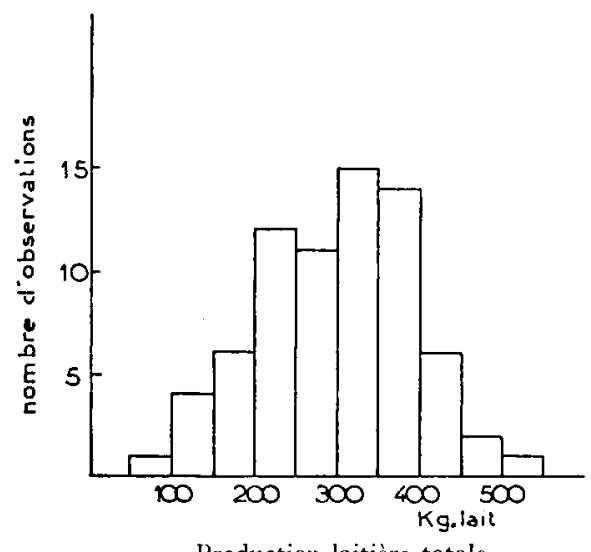

Production laitière totale

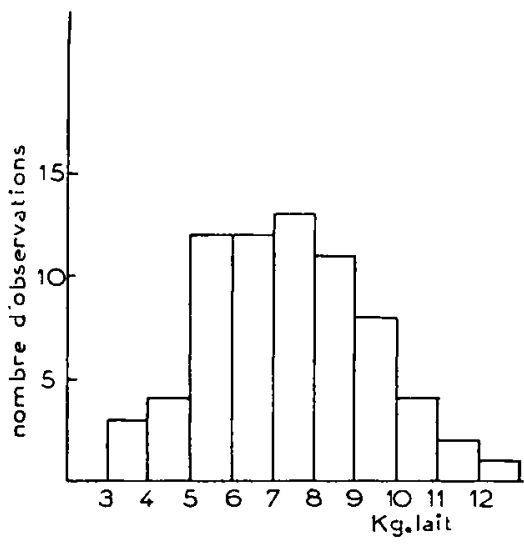

Maximum journalier

FIG. I. -- Distribution des productions laitières des truies.

\section{2o La courbe de lactation.}

On trouvera à la figure 2 la courbe moyenne établie à partir des résultats des 7 I lactations que nous avons suivies. Cette courbe est assez semblable à celle trouvée par d'autres chercheurs, mais il nous parait difficile cependant de la considérer comme courbe type de lactation de la truie. Il ne semble pas, en effet, qu'il y ait un type unique de courbe de lactation chez la truie, mais plusieurs types assez différents dont la résultante serait la courbe que nous présentons à la figure 2 .

Aussi croyons-nous préférable de donner à la figure 3 quelques exemples 


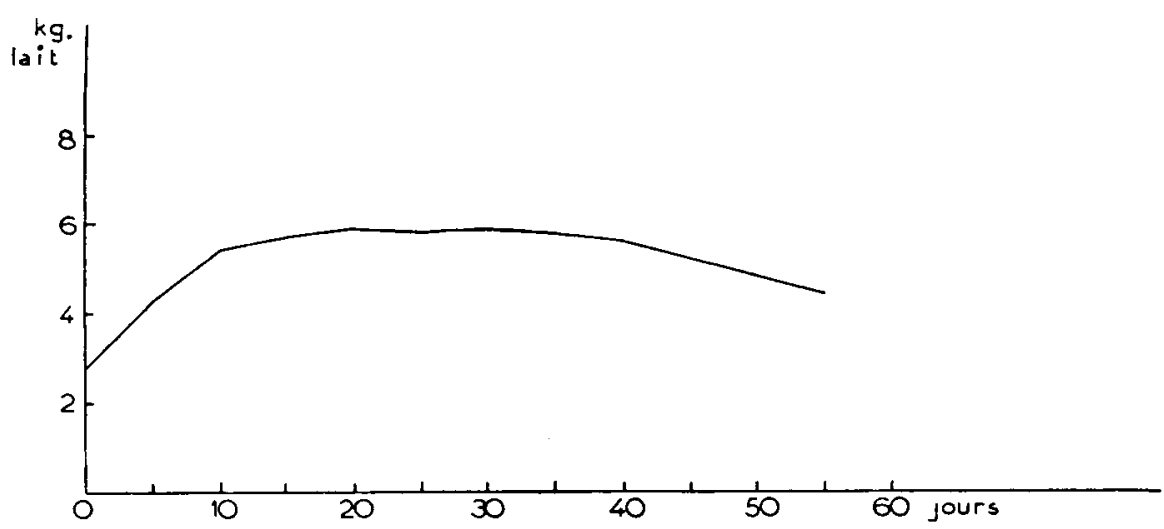

FIG. 2. - Courbe de lactation de la truie [moyenne de 7 I lactations individuelles].

de courbes de lactations individuelles s'apparentant aux types le plus souvent rencontrés ( 1$)$ :

- Les courbes du type A sont les plus fréquentes (5 I p. roo des cas) et correspondent à l'image de la lactation de la truie déjà décrite par des auteurs tels que Bonsma (I935), SMITH (I952), BARBER et BRAUdE (I955), Gili et Thomson (1956). Ces courbes présentent un maximum de la production journalière situé dans les premières semaines de lactation.

- Les courbes du type $B$ sont plus rares (2o p. Ioo des cas). Les lactations étudiées par BERGE et INDREBO (I953) sembleraient de ce type. Elles sont caractérisées par un maximum de production assez tardif.

- Les autres courbes (type $\mathrm{C}-29 \mathrm{p}$. Ioo des cas) n'appartiennent pas à un type bien défini. Le maximum de ces courbes est généralement peu ou pas apparent et le tracé souvent irrégulier. Les truies qui donnent de telles lactations sont souvent de mauvaises laitières ou des truies en début ou en fin de carrière.

\section{Remarques}

a) La forme de la courbe de lactation semble donc caractérisée par l'existence du maximum de la production journalière. Ce maximum survient cependant le plus souvent aux environs de la troisième semaine de lactation (en moyenne $24 \pm \mathrm{r} 8$ jours). Cette observation concorde avec celle de la plupart des auteurs (tableau II).

Il est intéressant de noter par ailleurs qu'il semble y avoir une liaison entre la valeur du maximum de production et le moment de la lactation auquel celui-ci survient, ainsi qu'il apparaît au tableau III.

(1) Il ne nous est pas encore possible, cependant, de préciser si l'existence de ces types est due à une caractéristique individuelle des truies ou à l'interaction des facteurs du "milieu ".

Annales de Zootechnie. - r958. 

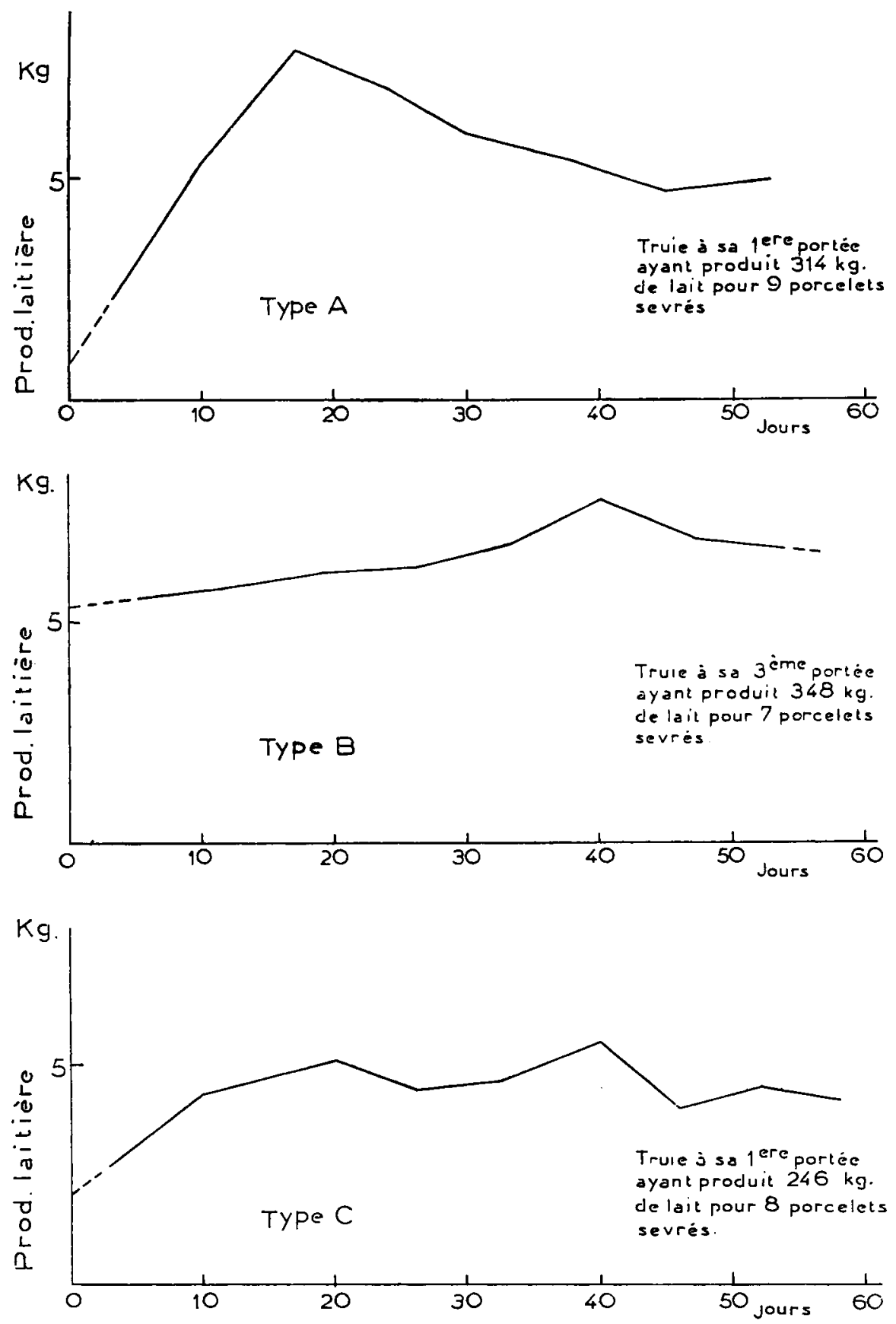

FIG. 3. - Exemples de courbes de lactation rencontrées dans la pratique. 
TABLEAU II

Maximum de production journalière des truies.

\begin{tabular}{|c|c|c|}
\hline Auteur & Année & Production maximum \\
\hline$\ldots \ldots \ldots \ldots$ & 1926 & $3^{\mathbf{e}}$ semaine \\
\hline Bonsma $\ldots \ldots \ldots \ldots \ldots \ldots \ldots \ldots \ldots \ldots$ & I 935 & $3^{\mathrm{e}}$ semaine \\
\hline Dschaparidse $\ldots \ldots \ldots \ldots \ldots \ldots \ldots \ldots$ & 1936 & $3^{\mathbf{e}}$ semaine \\
\hline Filmer $\ldots \ldots \ldots \ldots \ldots$ & 1949 & $6^{\mathbf{e}}$ semaine \\
\hline 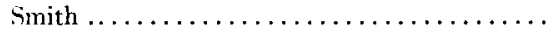 & $195^{2}$ & $4^{e}$ semaine \\
\hline Berge...$\ldots \ldots \ldots \ldots \ldots \ldots \ldots \ldots \ldots \ldots \ldots \ldots$ & 1953 & $5^{\mathrm{e}}$ semaine \\
\hline Clausen $\ldots \ldots \ldots \ldots \ldots \ldots \ldots \ldots \ldots \ldots \ldots$ & 1952 & $4^{\text {e semaine }}$ \\
\hline 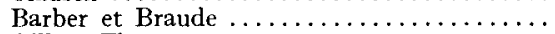 & 1955 & $3 \cdot 4^{\circ}$ semaine \\
\hline Gill et Thomson $\ldots \ldots \ldots \ldots \ldots \ldots \ldots \ldots \ldots$ & 1956 & $3^{\circ}$ semaine \\
\hline
\end{tabular}

TABLEAU III

Valeur du maximum de la production journalière suivant le moment auquel survient ce maximum.

\begin{tabular}{|c|c|c|c|c|c|c|}
\hline & \multicolumn{6}{|c|}{ Production maximum (j.) } \\
\hline 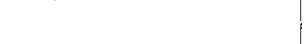 & $0 \cdot 10$ & II -20 & $2 I-30$ & $3 I-40$ & $4 I-50$ & $5 I \cdot 60$ \\
\hline $\begin{array}{l}\text { Nombre de cas ........ } \\
\text { Valeur du maximum }(\mathrm{kg} \\
\text { de lait } \ldots \ldots \ldots \ldots \ldots\end{array}$ & $\begin{array}{l}8 \\
6,47\end{array}$ & $\begin{array}{l}22 \\
8,07\end{array}$ & $\begin{array}{l}16 \\
7,21\end{array}$ & $\begin{array}{l}12 \\
7,20\end{array}$ & $\begin{array}{l}\mathrm{I} 2 \\
6,92\end{array}$ & I $33^{\circ}$ \\
\hline
\end{tabular}

Il existe une corrélation négative de coefficient $r=-0,270(n=62$, $\mathrm{P}=0,05$ ) entre ces deux variables (lorsque le maximum est postérieur au Io jour de lactation) : à partir de la deuxième semaine de lactation, le maximum tend à être d'autant moins important qu'il se présente plus tard. On peut montrer de la même façon que la production totale au cours de la lactation est aussi en corrélation négative $(r=-0,270$, $\mathbf{P}=0,05$ ) avec la date d'apparition de ce maximum. Ceci permet de penser que les truies dont les courbes de lactation présentent un maximum précoce, sont souvent celles qui produisent le plus de lait. Comme, par ailleurs, c'est au premier âge que le porcelet a le plus de peine à remplacer le lait maternel, ces deux raisons font penser qu'un maximum précoce provoque la meilleure croissance des porcelets.

b) La baisse de production qui suit le maximum de production est moins régulière que nel'indique la courbe moyenne de la figure 2 et il existe, à cet égard, d'assez grandes différences selon les truies. Cette baisse devient souvent plus accusée à partir de la $6^{\mathrm{e}}$ semaine, comme l'avaient déjà signalé BERGE (I952) et GIL, et THomson (I956).

c) La fin de la lactation surviendrait naturellement et suivant les truies, à des époques comprises entre la $6^{\mathrm{e}}$ et la $\mathrm{r}_{2} \mathrm{e}$ semaine de lactation. La huitième semaine étant le moment le plus courant du sevrage, c'est elle que nous avons prise dans notre étude pour terme de la lactation.

Nous donnons, toutefois, au tableau IV la répartition des produc- 
tions journalières que nous avons observées à ce stade. On constate qu'un certain nombre de truies (plus de $50 \mathrm{p}$. I00) ont encore des productions journalières élevées à ce moment.

\section{TABLEAU IV}

\begin{tabular}{|c|c|c|c|c|}
\hline Kg. de lait & $0 \cdot 2$ & $2 \cdot 4$ & $4 \cdot 6$ & 6 \\
\hline $\begin{array}{l}\text { Nombre de cas. } \\
\% \ldots \ldots \ldots \ldots \ldots\end{array}$ & $\begin{array}{r}8 \\
\text { II }\end{array}$ & $\begin{array}{l}20 \\
28\end{array}$ & $\begin{array}{l}26 \\
37\end{array}$ & $\begin{array}{l}17 \\
24\end{array}$ \\
\hline
\end{tabular}

\section{$3^{\circ}$ Production laitière à 21 jours et production totale.}

Les porcelets ne consomment jusqu'à trois semaines que du lait maternel et leur croissance est vraisemblablement le témoin plus ou moins fidèle de la quantité de lait qu'ils ont reçu jusqu'alors.

On a pu penser utiliser cette liaison pour apprécier l'aptitude laitière des truies en mesurant l'augmentation du poids des porcelets entre la naissance et $2 \mathrm{I}$ jours. Mais ceci sous-entend que la production de lait jusqu'à 2 I jours est elle-même en liaison très étroite avec la quantité de lait totale produite au cours de la lactation.

Nous avons voulu vérifier cette hypothèse en calculant la corrélation liant ces deux productions et nous avons trouvé :

$$
r=+0,856(n=7 \mathrm{I}, \mathrm{P}=0,00 \mathrm{I}) \text {. }
$$

On peut remarquer cependant que la production laitière à 3 semaines ne représente qu'environ le $\mathrm{r} / 3$ de la production totale :

$$
\frac{\text { P. } 2 \mathrm{I} \mathrm{j} .}{\text { P. totale }}=0,360 \pm 0,088 \text {. }
$$

On peut donc penser que la quantité totale de lait produite au cours de la lactation dépend davantage de la production des 5 dernières semaines à laquelle elle est d'ailleurs liée par un coefficient de corrélation :

$$
r=+0,95^{\circ}(n=7 \mathrm{I}, \mathrm{P}=0, \mathrm{ooI}) \text {. }
$$

Il existe toutefois une certaine correspondance entre les deux phases de la lactation (avant trois semaines et de trois semaines au sevrage), puisqu'on trouve entre les productions de ces deux périodes une corrélation $r=+0,665(n=7 \mathrm{I}, \mathrm{P}=0,0 \mathrm{I})$, ce qui traduit une régularité relative des courbes de lactation.

La production à 2 I jours donne une image du début de la lactation qui renseigne sur la rapidité avec laquelle le maximum de production est atteint. Elle peut aussi permettre, dans une certaine mesure, une classification des truies sur la base de la production laitiêre totale. 


\section{II. - VARIATIONS DE LA PRODUCTION LAITIERE}

Les facteurs qui font varier quantitativement la production laitière des truies sont nombreux. Nous avons retenu plus spécialement quelquesuns d'entre eux.

\section{1 o Le nombre de porcelets des portées.}

Bonsma (I935) avait déjà remarqué que les truies donnant le plus de lait étaient celles qui avaient les portées les plus nombreuses.

Cette observation, également formulée par BERGE (I953), se retrouve aussi dans la figure 4 où nous donnons les variations de la production avec le nombre de porcelets des portées.

Nous avons pu, à ce sujet, calculer les coefficients de corrélation suivants :

- Entre le nombre de porcelets à 3 semaines et la quantité de lait produite jusqu'à la même époque.

$$
r=+0,607(\mathrm{P}=0,0 \mathrm{I})
$$

- Entre le nombre de porcelets sevrés et la quantité totale de lait produite.

$$
r=+0,72 I\left({ }^{1}\right)(\mathrm{P}=0, \mathrm{OI})
$$

- Entre le nombre de porcelets à la naissance et la quantité totale de lait produite.

$$
r=+0,35 \mathrm{I}(\mathrm{P}=0, \mathrm{OI})
$$

Les deux premières corrélations montrent que pour une truie donnée la quantité de lait produite est liée au nombre de porcelets qu'elle élève dans sa portée : plus une truie nourrit de porcelets et plus elle produit de lait ${ }^{(2)}$. Ceci s'explique par le fait que le nombre de tétines productives chez la truie est généralement égal au nombre de porcelets (jusqu'à concurrence du nombre de tétines fonctionnelles).

Par contre, la liaison entre la production laitière et la prolificité des truies n'est qu'apparente, car la corrélation partielle qui lie ces deux variables est nulle :

$$
r=+ \text { o,org }(n=7 \mathrm{I})
$$

Ceci montre que ce n'est pas le nombre de porcelets à la naissance, mais le nombre de porcelets élevés qui est 1'un des facteurs déterminants de la production laitière. Cette observation confirme celle de RoEz (1932).

(1) Bonsma (r935) avait trouvé pour cette même corrélation $r=+0,586$.

(2) On pourrait aussi envisager l'hypothèse selon laquelle ce serait l'aptitude laitière de la truie qui conditionne le nombre de porcelets élevés et sevrés dans une portée ; cet intéressant problème sera étudié ultérieurement. 


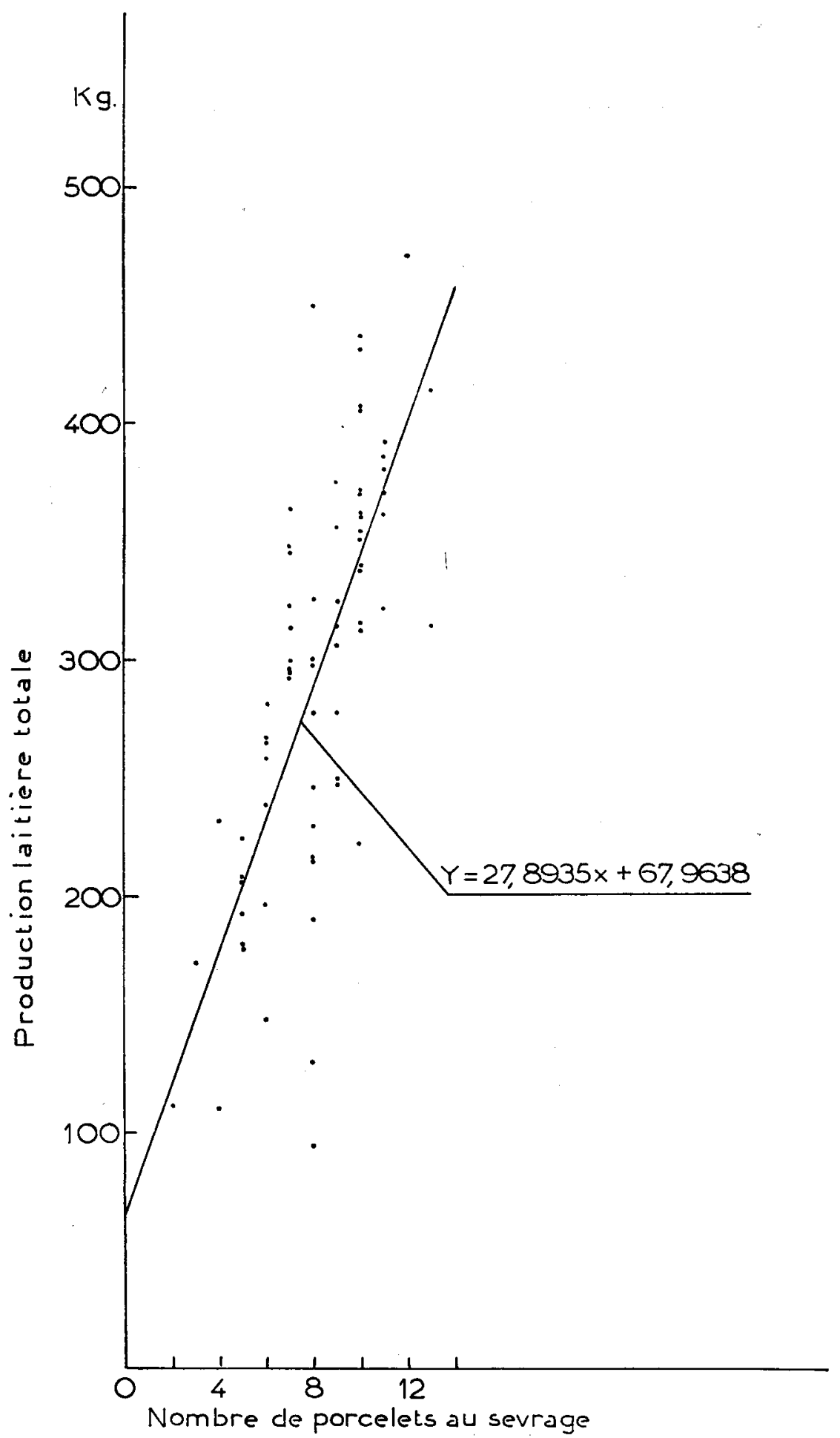

FIG. 4. - Variation de la production laitière en fonction du nombre de porcelets [au sevrage]. 
On peut illustrer ce phénomène en groupant les truies qui ont sevré sensiblement un même nombre de porcelets (8-9), après en avoir mis au monde des nombres différents (de 4 à $r_{4}$ ) : on constate bien qu'il n'y a pas de corrélation entre la production laitière et le nombre de porcelets à la naissance (tableau $\mathrm{V}$ ).

\section{Tableau V}

\section{Production laitière de truies de prolificité différente} mais ayant élevé le même nombre de porcelets.

\begin{tabular}{|c|c|c|c|c|}
\hline $\begin{array}{l}\text { No truie } \\
\text { No portée } \\
\end{array}$ & $\begin{array}{l}\text { Nb. de porcelets } \\
\text { à la naissance }\end{array}$ & $\begin{array}{l}\text { Nb. de porcelets } \\
\text { à } 2 \text { I j. }\end{array}$ & $\begin{array}{l}\text { Nb. de porcelets } \\
\text { sevrés }\end{array}$ & $\begin{array}{l}\text { Production } \\
\text { laitière }(\mathrm{kg}) \\
\end{array}$ \\
\hline $696 / 3 \ldots \ldots \ldots \ldots \ldots \ldots$ & 4 & $8(\mathbf{1})$ & 8 & 215 \\
\hline I $230 /$ I $\ldots \ldots \ldots \ldots \ldots \ldots \ldots$ & 4 & $8(\mathbf{1})$ & 8 & 297 \\
\hline $086 / 4 \ldots \ldots \ldots \ldots \ldots \ldots \ldots \ldots$ & 4 & $9(1)$ & 9 & 324 \\
\hline $256 / 3 \ldots \ldots \ldots \ldots \ldots \ldots \ldots$ & 8 & 8 & 8 & 325 \\
\hline $104 \mathrm{r} / \mathrm{I} \ldots \ldots \ldots \ldots \ldots \ldots \ldots$ & 9 & 8 & 8 & 246 \\
\hline $613 / 5 \ldots \ldots \ldots \ldots \ldots \ldots \ldots$ & Io & 9 & 9 & 247 \\
\hline $614 / 4 \ldots \ldots \ldots \ldots \ldots \ldots \ldots$ & Io & 9 & 9 & 375 \\
\hline $12 / 2 \ldots \ldots \ldots \ldots \ldots \ldots \ldots \ldots$ & I I & 8 & 8 & 449 \\
\hline $456 / 3 \ldots \ldots \ldots \ldots \ldots \ldots \ldots$ & I I & 9 & 9 & 277 \\
\hline $\operatorname{IIII} / 1 \ldots \ldots \ldots \ldots \ldots \ldots \ldots$ & II & 8 & 8 & I89 \\
\hline $885 / 5 \ldots \ldots \ldots \ldots \ldots \ldots \ldots$ & $\mathrm{I}_{4}$ & 8 & 8 & 277 \\
\hline $086 / 6 \ldots \ldots \ldots \ldots \ldots \ldots \ldots$ & 15 & 9 & 9 & 249 \\
\hline
\end{tabular}

Corrélation entre nombre de porcelets à la naissance et production laitière totale $r=+0,13$ non significatif.

Il faut noter, par ailleurs, comme l'ont fait remarquer Bonsma (I935) et BERGE (I953), que la production laitière et le nombre de porcelets ne varient pas proportionnellement : lorsque le nombre de porcelets croît, la quantité de lait reçue par chaque porcelet diminue (fig. 5).

Nous avons trouvé, en effet, une corrélation négative $r=-0,306$ ( $n=7 \mathrm{I}, \mathrm{P}=\mathrm{o}, \mathrm{or}$ ) entre la quantité de lait consommée par porcelet et le nombre de porcelets.

\section{go Le numéro d'ordre de la lactation.}

Bonsma (I935), Dschaparidse (I936), Donald (I937), Smith (I950), BERGE (I953) et LoDGE (I957) avaient remarqué que les truies donnaient généralement moins de lait aux premières lactations qu'aux suivantes (tableau VI).

Les résultats que nous avons obtenus sur ce point sont rapportés au tableau VII.

Seuls les résultats concernant les première et deuxième lactations sont significativement différents $(t=2,59, \mathrm{P}=0,0 \mathrm{I})$. Cette observation concorde avec celle de Bonsma (I935).

(1) Le nombre de porcelets a été complété à 8 ou 9 quelques jours après la mise-bas, par prélc̀vement sur des portées excédentaires. 


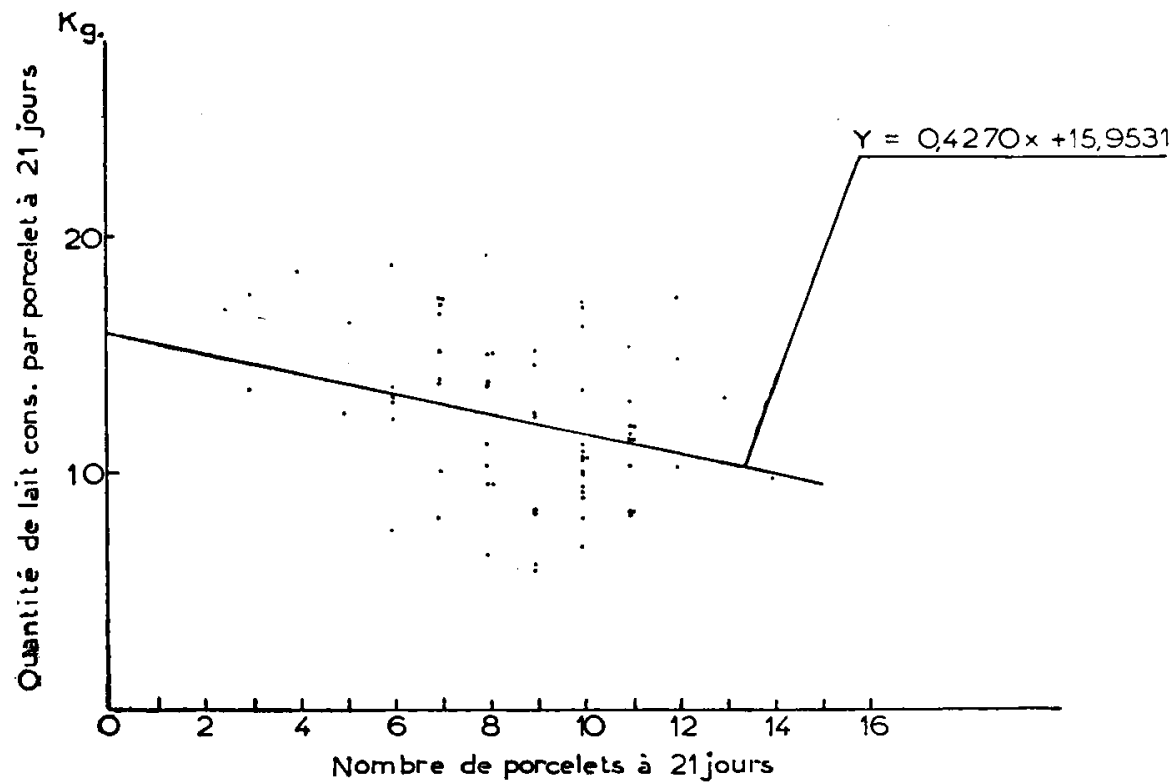

FIG. 5. - Quantité de lait consommée par porcelet en fonction dn nombre de porcelets [à 2 I jours].

\section{TABIEAU VI}

Variations de la production laitière avec le numéro d'ordre de la lactation.

$\begin{gathered}\text { Numéro } \\ \text { de la lactation } \\ m\end{gathered} \quad$
$\begin{gathered}\text { Production laitière } \\ \text { moyenne }\end{gathered}$

\section{TABLEAU VII}

Variations de la production laitière avec le numéro d'ordre de la lactation (C.N.R.Z.)

$N^{\circ}$ de la lactation

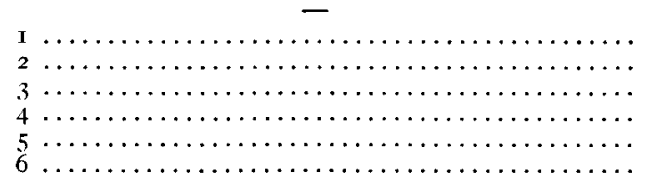

Nombre Production de lactations ( $\left.{ }^{1}\right)$ laitière $(\mathrm{kg})$

$\begin{array}{rrr}-53 & & 264 \\ 17 & & 337 \\ 14 & & 316 \\ 8 & & 309 \\ 6 & & 251 \\ 3 & & 276\end{array}$

On voit que la production laitière semble suivre l'évolution suivante avec 1'âge : assez forte augmentation $(+28 \mathrm{p}$. Ioo) entre la première et la

(1) Il ne s'agit pas entièrement ici de lactations consécutives enregistrées sur les mêmes truies. 
deuxième lactation, puis stabilité jusqu'à la quatrième lactation. I1 semblerait ensuite que la production baisse à partir de la cinquième lactation.

On peut rapprocher cette évolution soit de l'augmentation du poids de l'animal avec l'âge, phénomène généralement observé chez les bovins, soit des variations du nombre de porcelets au cours des portées successives, comme en témoigne le tableau VIII.

\begin{tabular}{|c|c|c|c|c|c|c|}
\hline & TABLE & $\mathrm{J} \mathrm{VI}$ & & & & \\
\hline No de la lactation & I & 2 & 3 & 4 & 5 & 6 \\
\hline $\begin{array}{l}\text { roduction laitière ( } \mathrm{kg} \text { ) .......... } \\
\text { oids de la truie (a la mise-bas) } \mathrm{kg} \text {. } \\
\text { lombre de porcelets au sevrage (moy.) }\end{array}$ & $\begin{array}{l}2 \overline{264} \\
184 \\
7,8\end{array}$ & $\begin{array}{l}-\overline{3} \\
219 \\
219 \\
9,1\end{array}$ & $\begin{array}{l}3 \overline{16} \\
258 \\
8,1\end{array}$ & $\begin{array}{l}. \overline{309} \\
265 \\
8,0\end{array}$ & $\begin{array}{l}251 \\
302 \\
8,1\end{array}$ & $\begin{array}{c}\mathbf{1} 76 \\
\mathbf{1} 75 \\
7,7\end{array}$ \\
\hline
\end{tabular}

On remarque, en particulier, que les variations du nombre de porcelets dans les différentes portées peuvent rendre compte des variations de la production laitière.

\section{$3^{\circ}$ Le poids de la truie.}

Bonsma (I935) avait mis en évidence une corrélation négative $r=-0,365$ entre le poids des truies et leur production laitière.

GROUDEV (I956) croit, par contre à une influence positive du poids des truies sur la production laitière, mais peut-être cela provient-il de ce que cet auteur ne semble pas avoir dissocié les facteurs poids des truies et nombre de porcelets qui varient parfois dans le même sens.

Selon nous, sauf peut-être pour le cas de la première portée, le poids propre des truies ne semble pas influer sur la production laitière :

- Corrélation entre le poids de la truie à la saillie et la production laitière :

$$
\mathrm{r}=-0,0 \mathrm{I} 8 \quad(n=66, \text { non significatif })
$$

- Corrélation entre le poids de la truie à la mise-bas et la production laitière :

$$
r=+0,085 \quad(n=55, \text { non significatif). }
$$

Nous avons trouvé, par contre, une certaine corrélation, $r=+0,399$ $(n=5 \mathrm{I}, \mathrm{P}=0, \mathrm{OI})$ entre le gain de poids de la truie pendant la gestation et la production laitière. On peut donc penser qu'une petite truie peut être aussi laitière qu'une grosse à condition d'être bien nourrie et de prendre du poids pendant sa gestation (fig. 6).

De la même façon, nous avons trouvé une corrélation très nette $r=+0,545(n=6 \mathrm{I}, \mathrm{P}=0,0 \mathrm{r})$ entre la perte de poids des truies pendant la lactation et les quantités de lait produites. Ceci confirme que les bonnes laitières sont souvent les truies qui perdent le plus de poids pendant la lactation (fig. 7). 


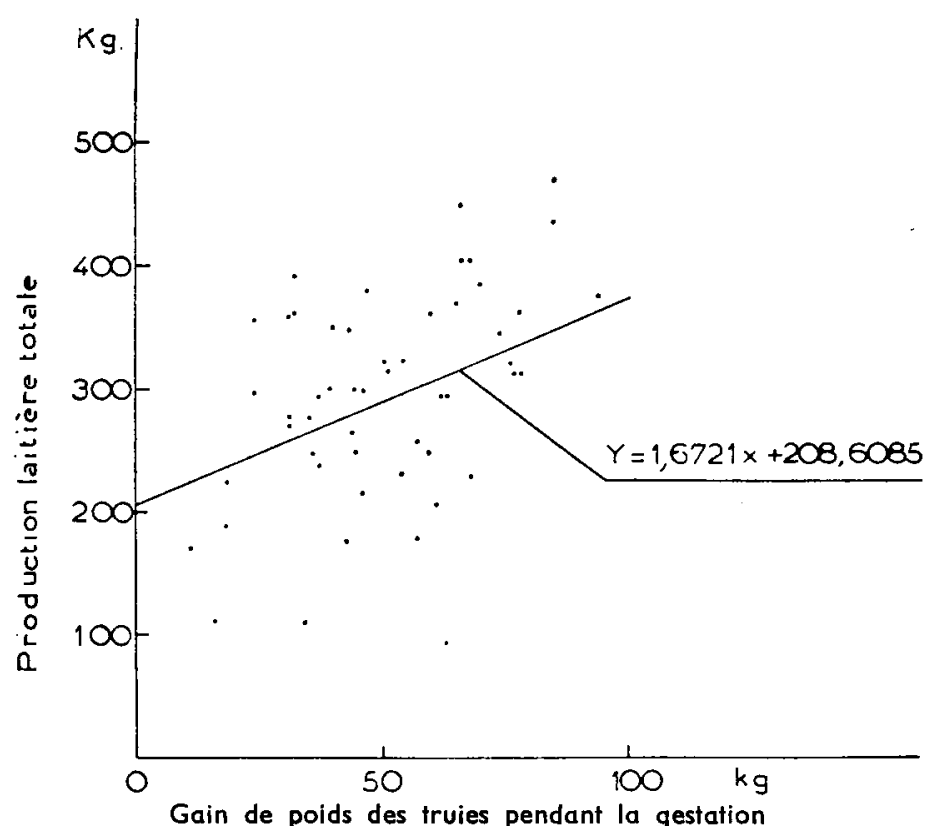

FIG. 6. - Influence du gain de poids pendant la gestation sur la production laitière des truies.

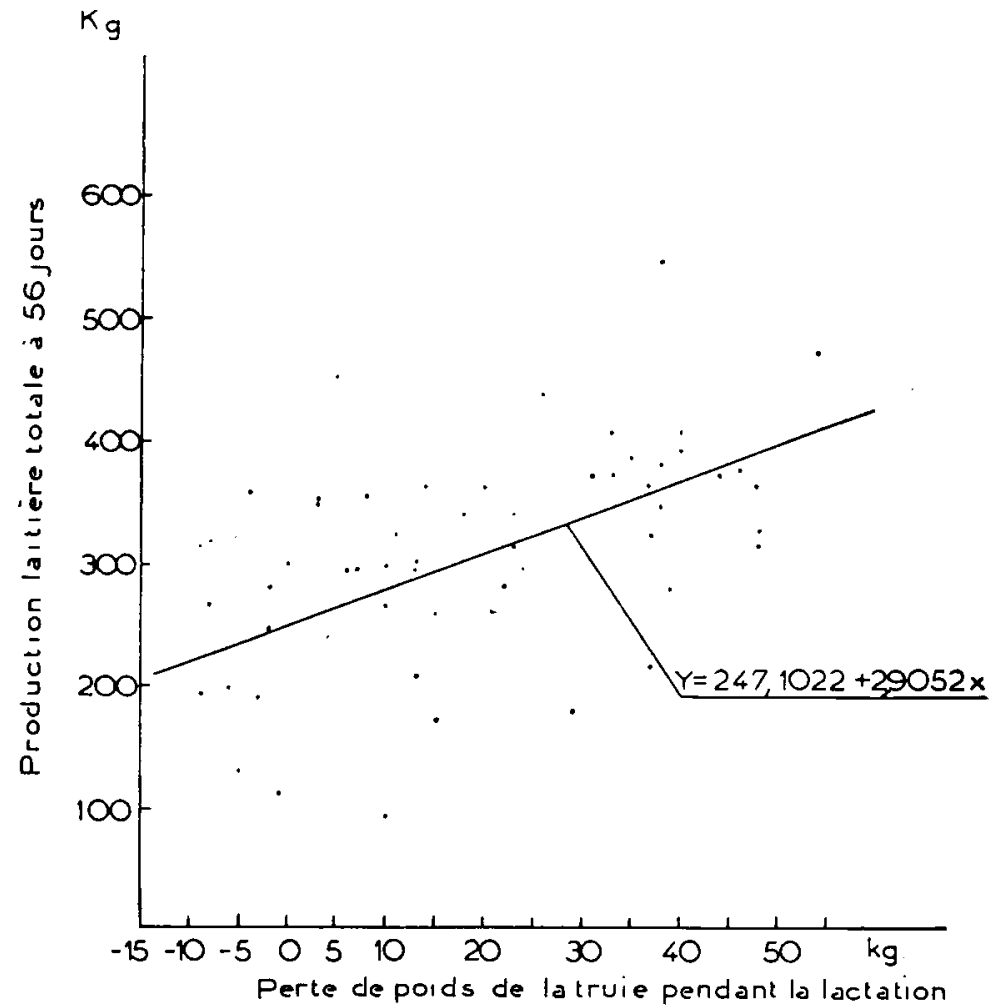

FIG. 7. - Production laitière et perte de poids de la truic pendant la lactation. 


\section{La consommation alimentaire.}

Il semble que la production laitière des truies soit fortement liée aux quantités d'aliments ingérées par la truie pendant sa lactation. Ce point a fait l'objet d'une étude particulière, (SALmon-LEGAGNEUR, I957), dont nous résumons ici les conclusions :

I $^{\circ}$ La production laitière journalière est sous la dépendance de la consommation journalière de la truie.

$2^{\circ}$ Il paraît possible d'améliorer les conditions de production laitière de la truie en stimulant (par apport d'aliments appétents) la consommation d'aliments de cette dernière.

D'autres études sont actuellement en cours sur cette importante question.

\section{5o La race.}

Il est logique de penser que, comme pour les autres espèces, il existe chez le porc des races dont les truies sont plus ou moins laitières. Il paraît difficile, cependant, de considérer comme représentatifs de la race les résultats fragmentaires obtenus jusqu'à ce jour.

A titre indicatif, nous donnons toutefois au tableau IX les plus récents résultats se rapportant à différentes races.

\section{TABLEAU IX}

\section{Productions laitières de différentes races porcines.}

\begin{tabular}{|c|c|c|c|}
\hline Race & $\begin{array}{l}\text { Nombre } \\
\text { de } \\
\text { lactations }\end{array}$ & $\begin{array}{l}\text { Production laitière } \\
\text { journalière moyenne } \\
(\mathrm{kg})\end{array}$ & Auteur \\
\hline - & - & -3 & - \\
\hline $\begin{array}{l}\text { Poland china } \\
\text { Duroc Jersey }\end{array}, \ldots \ldots \ldots \ldots$ & 43 & 4,5 & Wells et Beeson (1940). \\
\hline Middle White (Japon) ....... & 9 & $\therefore, 2$ & Niwa et Ito (195I). \\
\hline Berkjala (Philipp.) . ......... & 8 & I,t & Ruma (195I). \\
\hline Berkshire $(\mathrm{N}, \mathrm{Z} .)^{\prime}$. & I 5 & 5,4 & Smith (1952). \\
\hline Landrace Danoise ........... & 13 & $7.5(1)$ & Clausen (1952). \\
\hline Landrace Norvégienne ....... & 28 & 7,5 & Berge et Indrebo (1953). \\
\hline Morava $\ldots \ldots \ldots \ldots \ldots \ldots$ & 24 & 2,2 & Lalevic (I953). \\
\hline 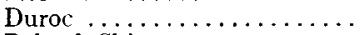 & 12 & 2,4 & Allen et Baker (1955). \\
\hline Poland China ............. & 3 & 2,9 & " \\
\hline Wessex-Saddle back ........ & 12 & 7,9 & Lodge (1957). \\
\hline Large White ............. & 3 & 6,2 & Barber et Braude (1955). \\
\hline$n \quad n \quad \ldots \ldots \ldots \ldots \ldots$ & 6 & 4,9 & Gill et Thomson (1956). \\
\hline$n$ & $7 \mathrm{I}$ & 5,3 & Salmon-Legagneur (1957) \\
\hline
\end{tabular}

\section{(io Variations d'origine anatomique.}

I.es tétines antérieures de la mamelle des truies produisent plus de lait que les postérieures (on sait que c'est là une des raisons invoquées pour expliquer le choix de ces tétines par les porcelets dans les jours qui suivent la mise-bas). Nous avons pu vérifier ce phénomène, déjà signalé par DONALD (I937) et par BARBER et BRAUdE (I955), en pesant les quan-

(1) Moyenne sur 6 semaines de lactation seulement. 
tités de lait que 1'on peut recueillir par traite après injection intra-veineuse d'ocytocine. Nous avons trouvé sur 63 couples de mesures :

$\begin{array}{lr} & \text { Production moyenne par tétine } \\ \text { Tétines antérieures (2 paires) } & 34,2 \mathrm{~g} . \\ \text { Tétines postérieures (3 paires) } & \mathrm{I} 9,0 \mathrm{~g} \text {. }\end{array}$

Par contre, le nombre de tétines de la truie (ligne de mamelle) ne semble pas avoir une grande importance : il n'y a pas de corrélation apparente entre le nombre de tétines et la production laitière des truies $(r=+0,054$, non significatif). Ceci est sans doute lié au fait que nous n'avons pas trouvé davantage de corrélation entre le nombre de tétines et le nombre de porcelets à la mise-bas : ( $r=+0$, I I9, non significatif).

\section{III. - PRODUCTION LAITIÈRE DE LA TRUIE ET SÉLECTION.}

I,a sélection sur la valeur laitière semble possible chez le porc. En dépit de l'imprécision de la méthode de mesure, nous avons pu en effet trouver, sur les animaux que nous avons contrôlés une répétabilité brute (lactations non corrigées) de 0,370 entre les lactations successives.

Cette valeur est comparable à celle que l'on obtient chez les bovins. Par ailleurs, la variabilité de la production laitière des truies semble assez forte (fig. I).

Mais la mesure de la production laitière des truies est délicate et difficilement généralisable dans la pratique. Ia connaissance de la production laitière en elle-même des truies importe d'ailleurs peu au sélectionneur lorsque son but est de produire des portées nombreuses et lourdes. Comme 1'a souligné FÉVRIER (1956), la production laitière constitue l'un des facteurs d'obtention de portées lourdes au sevrage et il paraît tout aussi raisonnable de sélectionner directement sur le but recherché, le poids des porcelets, qui constitue d'ailleurs un caractère plus facile à mesurer.

Il peut être utile cependant, à des fins expérimentales, de se livrer à des mesures plus précises et il est bon, dans ce cas, de connaître la liaison qui existe entre la production laitière des truies et le poids des porcelets. Certains auteurs, tels que I,EROY (I937) et NAvRATIL (I955), utilisent, en effet, cette dernière donnée pour apprécier la valeur laitière des truies.

Il existe, en effet, une corrélation assez étroite entre le gain de poids des porcelets (entre la naissance et 2I jours, âge auquel le lait maternel reste encore leur aliment presque exclusif) et la quantité de lait qu'ils reçoivent. Nous avons trouvé entre ces deux caractères le coefficient de corrélation le suivant:

$$
r=+0,736(n=71, \mathrm{P}=0,0 \mathrm{I}) \quad \text { (fig. 8). }
$$

En conséquence, le poids de la portée (à 2 I jours ou à 56 jours) apparaît comme la donnée facilement mesurable la plus en rapport avec la production laitière (fig. 9 et Io). 
- Corrélation entre la production laitière à 2I jours et le poids de la portée à cette date :

$$
r=+0,830 \quad(n=7 \mathrm{I}, \mathrm{P}=0, \mathrm{oor})
$$

- Corrélation entre la production laitière totale (à 56 jours) et le poids de la portée au sevrage :

$$
r=+0,857(n=7 \mathrm{I}, \mathrm{P}=0,00 \mathrm{I}) \text {. }
$$

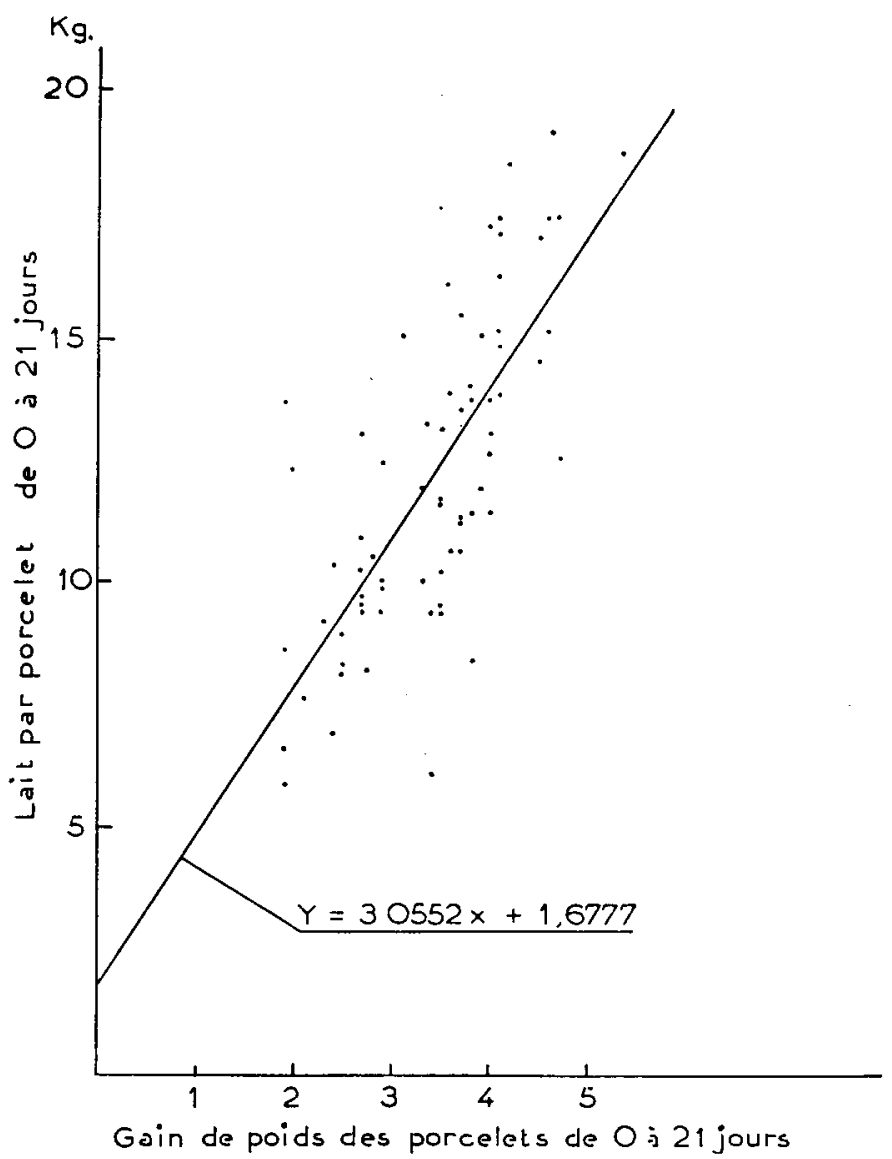

Fig. 8. - Croissance des porcelets en fonction des quantités de lait qu'ils consomment.

Cette dernière corrélation, très élevée, montre bien la valeur du poids total de la portée au sevrage comme témoin de la production laitière de la truie. Nous donnons, à ce sujet, au tableau X une comparaison des différents critères que l'on aurait pu penser utiliser dans ce domaine.

La sélection sur le poids de la portée au sevrage parait donc rationnelle et semble un moyen sûr d'améliorer ipso-facto la production laitière des truies. 


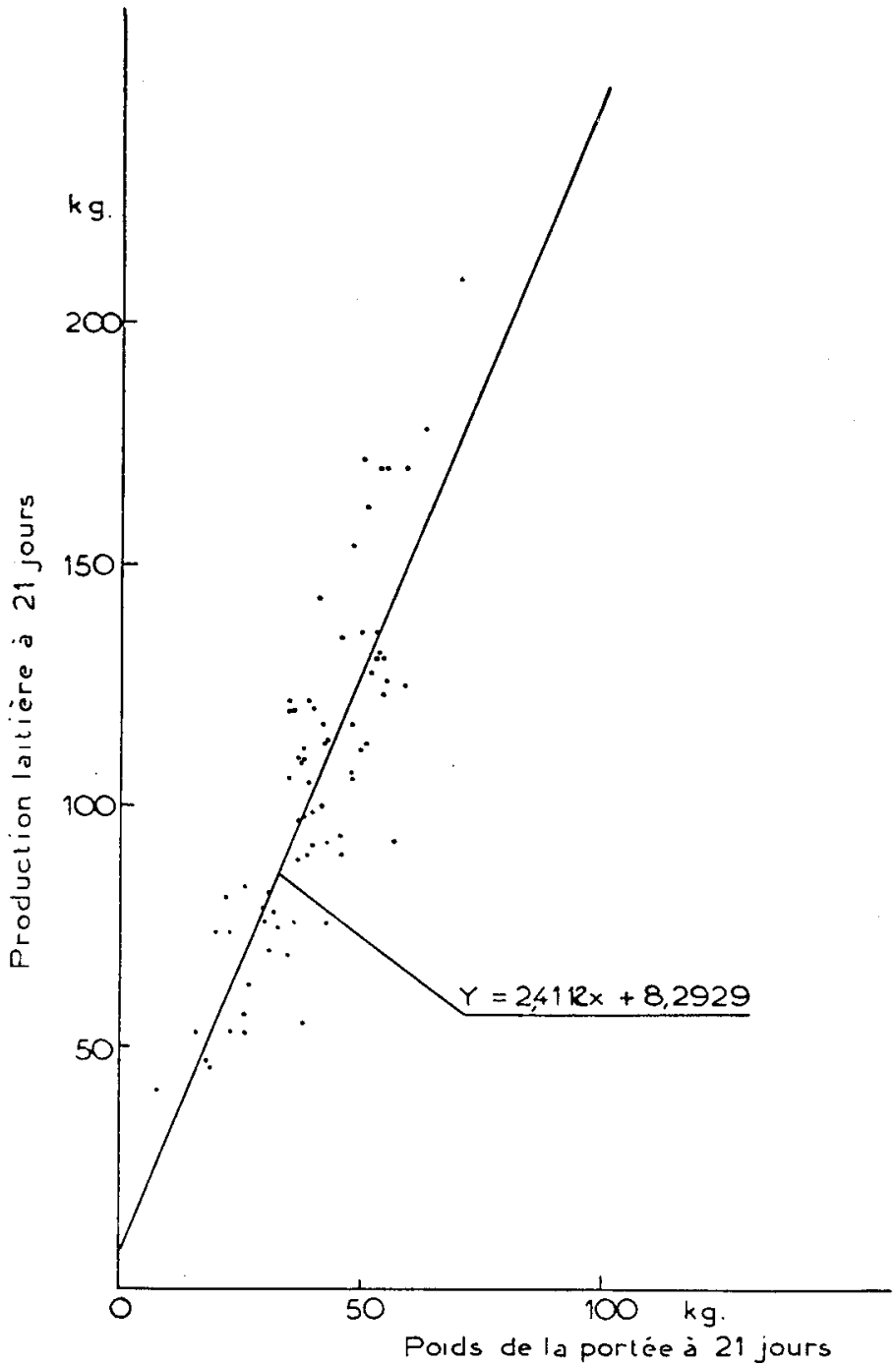

FIG. 9. - Poids de la portée et production laitière des truies [à 2I jours].

\section{TABLEAU $\mathrm{X}$}

Coefficients de corrélation liant la production laitière des truies à différents caractères.

Corrélation entre la production laitière totale (lactations de 56 jours) et
Coefficient de corrélation de signification

Poids de la portée au sevrage.

Poids de la portée à 21 jours

$\begin{array}{ll}+0,857\left(^{1}\right) & P=0,001 \\ +0,774 & P=0,001 \\ +0,511 & P=0,01 \\ +0,721\left(^{2}\right) & P=0,01\end{array}$

Nombre de porcelets sevrés

(1) Cette valeur du coefficient de corrélation est significativement supérieure à celle obtenue pour le poids moyen des porcelets $(\mathrm{P}=0,0 \mathrm{I})$.

(2) Il paraît intéressant de noter que la valeur de ce coefficient de corrélation semble du même ordre 


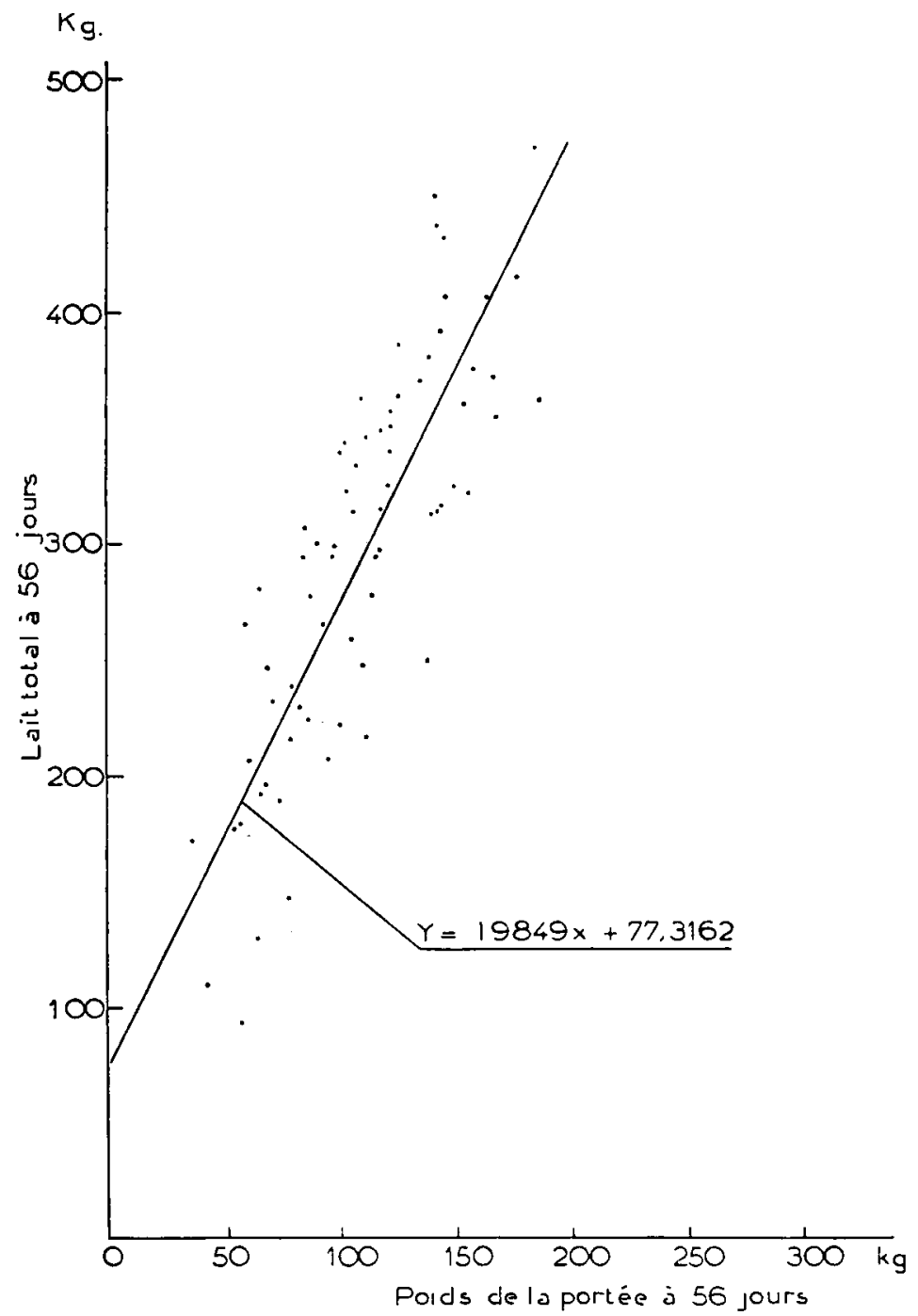

FIG. 10. - Poids de la portíe et production laitière des truies [au sevrage].

Un point reste intéressant à signaler : il semble que la forme de la courbe de lactation ne soit pas sans importance pour la croissance des porcelets. On peut penser que, toutes conditions étant égales par ailleurs, les truies qui ont la plus forte production au début de la lactation sont aussi celles qui produisent les portées les plus lourdes au sevrage. Ce phénomène est illustré par le tableau XI et la figure II.

que celle obtenue pour le poids de la portée à 2r jours. Il pourrait donc être aussi fondé de sélectionner sur le nombre de porcelets au sevrage que sur le poids de la portée à 3 semaines. Nous nous proposons de vérifier par la suite cette hypothèse. 


\section{TABLEAU XI}

Variations du poids de la portée au sevrage pour des truies ayant des nombres très voisins de porcelets et sensiblement la même production totale, mais dont la production à $2 \mathrm{I}$ jours varie.

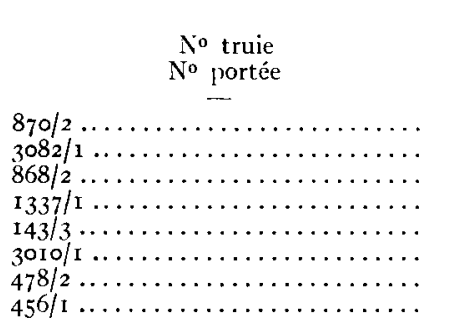

Nombre de porcelets Production laitière Poids de la portée à $2 \mathrm{I} \mathrm{j}$. au sevrage ’̀ $2 \mathrm{I} \mathrm{j}$. au sevrage à $2 \mathrm{I}$ j. au sevrage

kg.lait

\begin{tabular}{|c|c|c|c|c|c|}
\hline- & - & - & - & - & - \\
\hline I I & I I & 186 & 361 & 52 & I 28 \\
\hline I I & IO & I68 & 354 & 59 & 125 \\
\hline IO & 10 & 167 & $37 \mathrm{I}$ & 46 & I 35 \\
\hline IO & IO & 154 & $3^{60}$ & 50 & I I 2 \\
\hline I I & I I & 148 & 370 & 54 & 126 \\
\hline ro & IO & I 35 & $37^{\circ}$ & 47 & II 7 \\
\hline IO & 9 & I 22 & 356 & 42 & 100 \\
\hline I I & Io & I 22 & $35^{\circ}$ & 43 & 93 \\
\hline
\end{tabular}

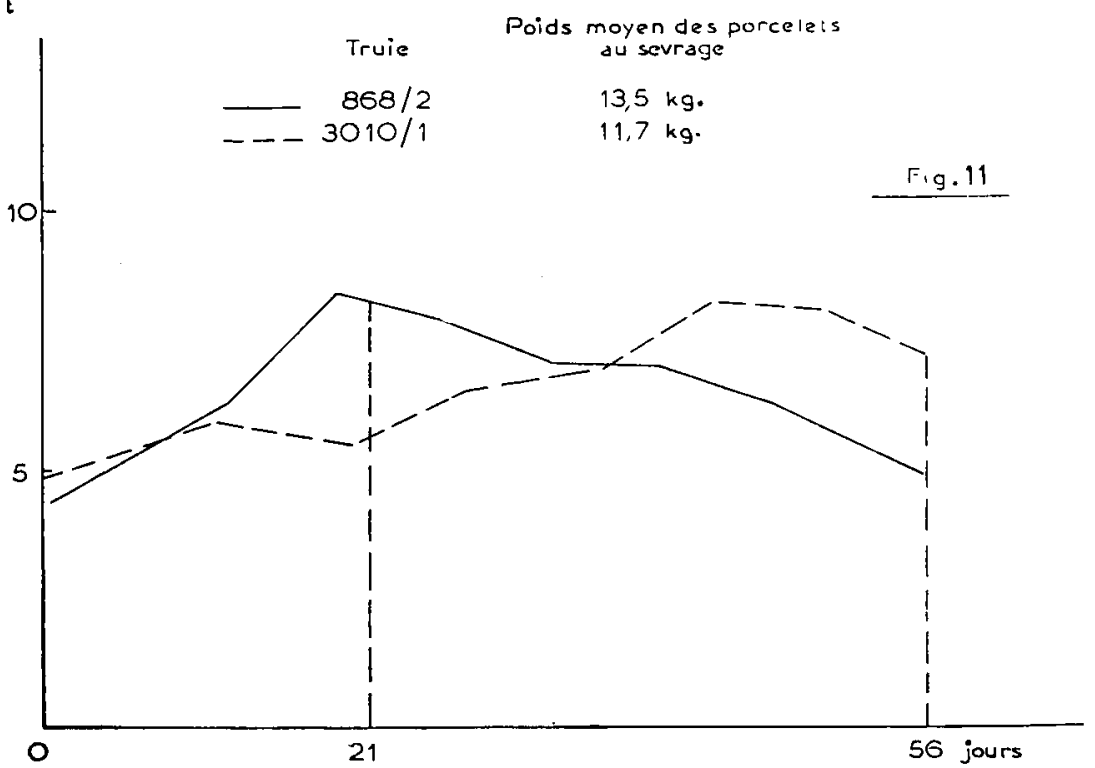

FIG. II. - Exemple de truies ayant eu, pour un même nombre, de porcelets des productions laitières identiques, mais dont les productions jusqu'à $2 \mathrm{r}$ jours [et la forme de la courbe de lactation] diffèrent.

Nous avons trouvé pour les 8 truies du tableau XI une corrélation entre la production laitière à 2 jours et le poids de la portée au sevrage $r=+0,833(n=8, \mathrm{P}=0,05)$.

De la même manière, on peut mettre en évidence sur l'ensemble des résultats $(n=7 \mathrm{r})$ une corrélation partielle (indépendante de la production laitière totale), peu significative il est vrai, entre la production laitière jusqu'à 2 I jours et le poids de la portée au sevrage $r=+0,203(\mathrm{P}=0,07)$.

Ceci montre l'intérêt d'un bon départ de lactation chez la truie. Comme nous l'indiquions au début de cette étude (cf. courbe de lactation), 
un maximum de production journalière précoce et élevé, même avec une moindre persistance pour le reste de la lactation, semble conditionner une bonne croissance des porcelets. Le poids de la portée à $2 \mathrm{I}$ jours pourrait alors, dans ce domaine, donner des renseignements utiles et servir de base à une étude plus approfondie du phénomène.

\section{RESUME}

7r lactations de truies ont été enregistrées après mesure de la production laitière par pesée des portées de porcelets avant et après les tétées. La production moyenne par truie a été de $5,3 \mathrm{~kg}$ de lait par jour, ou de $297 \pm 9 \mathrm{I} \mathrm{kg}$ de lait par lactation ( 56 jours).

L'étude de quelques facteurs de variation et de la production laitière a montré :

$I^{0}$ Que le nombre de porcelets élevés dans la portée était un facteur important de variation. Il existe une corrélation $r=+0,72 \mathrm{I}$ entre le nombre de porcelets de la portée et la quantité de lait produite au cours d'une lactation.

$2^{\circ}$ Que les truies produisaient généralement plus de lait à leur deuxième lactation (20 à $30 \mathrm{p}$. Ioo de plus qu'à la première lactation), mais qu'il $\mathrm{y}$ avait peu de variations apparentes ensuite.

$3^{\circ}$ Que le poids propre de la truie influait peu, mais que 1'augmentation du poids de la truie pendant la gestation pouvait jouer un certain rôle sur la production.

$4^{\circ}$ Que l'appétit de la truie influait probablement sur la production laitière.

$5^{\circ}$ Que la race Large White semblait, compte tenu des résultats actuels, se placer parmi les races étudiées ayant une assez bonne production laitière.

Nous avons pu montrer, par ailleurs, qu'il semblait possible d'apprécier la valeur laitière des truies en s'appuyant sur le poids total de la portée au sevrage (corrélation avec la production laitière totale : $r=+0,857$ ) et sur le poids de la portée à 2 I jours. L'intérêt essentiel de cette dernière mesure est de renseigner sur la forme des courbes de lactation ; celle-ci peut, en effet, avoir une influence sur la croissance des porcelets, ce qui sera vérifié ultérieurement.

\section{RÉFÉRENCES BIBLIOGRAPHIQUES}

AlLEN (A. D.), BAKER (R. O.), LASLEY (J. F.). - Milk production of sows as correlated with the performance of pigs. J. Anim. Science, 14, (4), II-74, I955.

Barber (R. S.), Braude (R.), Mitcheli (K. G.). - Studies on milk production of Large White pigs. J. Agric. Sci., $\mathbf{4 6}$ (I), 97-II8, I955. 
Berge (S.), Indrebo ('T.). - Mjolkeproduksjon hos purker. Medl. Norges Landbrukshogskole, 33, 389-424, I953.

Bonsma (F. N.). - Milkproduction in large black sows. So African J. of Sci., 32, 360, I935.

CARLYLE (W. L.). - The food requirements of pigs from birth to maturity. Wis. Bull. Agric. Exp. Sta., no I04, p. 8, I903.

ClaUsen (H.). - Investigations concerning the sows milk yield (Communication personnelle), I952.

DONALD (H. P.). - The milk consumption and growth of suckling pigs. $E m p$. J. Exp. Agric., 5, 349-360, I937.

Dschaparidse, (D.). - Die Milchleistung der schweine. Z. Zucht. B., 34, 349-57, I936.

FÉvrieR (R.). - Quelques réflexions sur le contrôle des aptitudes chez le porc. Journées AFZ sur le contrôle des aptitudes. Paris, 1956.

Filmer (J. F.). - Observations on the milk yield sows. Rep. N. Z. Dept. Agric., I $948-49,87$, I 949 .

GiLI (J.C.), Thompson (W.). - Effect of environmental temp. on suckling pigs and a study of the milk yield of the sows. J. Agric. Sci., 47, 324, I956.

GOHREN (T. von). - Landwirtsch. Versuchs-Stat., \%, S 35I (cité par BRAUDE), I865.

Groudev (D. I.). - Influence de la taille des truies pour l'élevage du porc. Elevage U. R. S. S., 9, 52, I956.

Hempes (K.).- - Ueber die Milcheistung der Sauen des veredelten Landschweins. Arbeiten der Deutschen Gesellschaft für Zucht. Heft 37, I9-40, I928.

IAALEVIC (D.). - Prilog poznavanjer mlecnosti svinje moravke Archpoljopr. Nauk., 6 (I3), I23, I953.

IEROY (A. M.). - Le Porc, p. 159. Hachette, 1937.

LODGE (G. A.). - Studies on yield and composition of sow's milk. Comm. $4^{\mathrm{e}}$ Congrès Intern. de Nutrition, Paris, I957.

Navratil (B.). - Comparaison de la production laitière des truies suivant le poids des porcelets les $2 \mathrm{I}^{\mathrm{e}}$ et $28^{\mathrm{e}}$ jours. Zivoc. Vjroba. Veter. Med., 1, $43-58$, I955.

Nrwa (T.), Ito (S.), Yokoyama (H.), Otrsuka (M.). - Studies on the milk secretion of the sow. Bull. Nat. Inst. Agric. Sci. Japan, Ser. G., 1, I35-50, I95I.

OLOFSSON (N. E.), LARSSON (S.). - Modersuggornas produktion och smagrisarnas utveckling. Centralanstalten Mdd 374. Husdjursavdelningen 68, I930.

RoEz (M.). - Untersuchungen über Milcheistung des Mangaleia schweines (cité par BONSMA), I932.

Ruma (M.). - The lactation of Berkjala sows. Philipp. Agric., 35, 217, I95I.

SAIMON-LEGAGNEUR (E.). - La mesure de la production laitière chez la truie. Ann. Zoot., 5 (2), 95-IIo, I956.

SALMON-LEGAGNEUR (E.). - Consommation alimentaire et production laitière chez la truie. Comm. $4^{\text {e }}$ Congrès Intern. de Nutrition. Paris, 1957.

SchmidT (J.), LAUPRECHT (E.). - Uber die milch der veredelten Landschweinsaüen. Zuchtungskunde, 1, 50-62, I926.

Smith (D. M.). - Milk production in the sows. Prac. N. Z. Soc. Anim. prod., roth. ann. conf. 89-90, I950.

Smith (D. M.). - Yield and composition of milk of new zel. Berkshire sows. N. Z. J. Sci. Technol., 34 (I), 65-76, I952.

Welis, (W.), Beeson (W. M.), BRAdy (D. E.). - Bull. Idaho Agr. Exp.Sta., 236, 9 (cité par BARBER et BRAUDE), I940.

WOHIBIER (W.). - Stoffwechselversuche zum Eiweissansatz bei saugenenden ferkelen. Biochemische Zeitschrift., 202, 29, I928. 